\title{
The safety and efficacy of sotalol in the management of acute atrial fibrillation: a retrospective case control study
}

\begin{abstract}
Objective: The European Society of Cardiology, American Heart Association and the American College of Cardiology guidelines on atrial fibrillation (AF) 2006 state that Sotalol should not be used in acute AF. We assessed the safety and efficacy of sotalol in acute AF when compared to other anti-arrhythmic drugs (ADD).
\end{abstract}

Methods: A single centre retrospective observational study on 300 patients admitted with acute AF over a 12 months period. Study drugs used were sotalol, amiodarone, flecainide, propafenone or disopyramide for rhythm control. Digoxin, beta blockers, verapamil, diltiazem were prescribed for rate control. Rates of cardioversion to sinus rhythm, readmission rates due to AF, all cause readmissions, mortality rates due to sudden cardiac death and all-cause mortality was recorded over a 2 year follow up period. For paired data, the Wilcoxon matched-pairs signed- ranks or paired t-test were used. For unpaired data, Fisher's exact test was used.

Results: 120 patients were discharged on sotalol. The mean total dose used was $169.2 \mathrm{mg}$ daily. Cardioversion to sinus rhythm on discharge occurred in $68 \%$ in the rhythm control group versus $42 \%$ for rate control group $(p<0.001)$. Sotalol had a significantly higher cardioversion rate regardless of the dose when compared to amiodarone $(p=0.036)$ however, there were similar readmission rates for AF. Four patients died acutely in hospital, none were on sotalol. Compared to all drugs sotalol had the lowest mortality rates $(p=0.001)$. Mortality rates were lower in patients who received the higher dose of sotalol; $7.4 \%$ for patients who received a total of $320 \mathrm{mg}$ daily versus $11.8 \%$ in those who received $160 \mathrm{mg}$ daily.

Conclusion: Sotalol is as safe and effective as other anti-arrhythmic drugs; in fact it was significantly more effective than amiodarone in this cohort. All AAD's demonstrated a significant improvement in cardioversion rates and a significantly lower mortality rate than rate controlling drugs.

Submitted: 20 May 2015; Accepted: 23 June 2016; Published online: 28 June 2016

Keywords: Atrial fibrillation - Anti-arrythmics - Rate limiting drugs - Cardioversion commentary

Atrial fibrillation (AF) is the most common cardiac arrhythmia and affects $1.5-2 \%$ of the population [1]. Over 6 million Europeans have $\mathrm{AF}$ and the prevalence is expected to double in the next 50 years [2].

$\mathrm{AF}$ is associated with increased risk of stroke, heart failure, impaired quality of life, reduced exercise tolerance, left ventricular systolic impairment and death. Subsequently there is significant cost implications associated $[1,2]$. Attempting to achieve sinus rhythm is therefore of importance.
Antonio L Aguilar-Shea*

Centro de Salud Puerta de Madrid, Spain *Author for correspondence: Tel.: +34918806699 antonio.aguilar@salud.madrid.org 
Sotalol possesses a mixture of Vaughn-Williams class II and class III effects. The combination of rate and rhythm control properties may improve the chance of early restoration to sinus rhythm in patients who are in acute AF. This may limit the tendency towards electrical and structural remodelling seen in $\mathrm{AF}$ and improve the chance of long term rhythm control [3].

The use of sotalol for recent AF (less than 48 hours) is a class III indication and not recommended in the American College of Cardiology (ACC) American Heart Association (AHA) European Society of Cardiology (ESC) 2006 guidelines for AF [4]. However, it is important to note that a lack of clinical trials formed the basis of the guidelines.

In the 2012 ESC AF guidelines, sotalol can be used for $\mathrm{AF}$ if there is minimal or no structural heart disease [1]. In the ACC/AHA/ Heart Rhythm Society 2014 AF guidelines there is a class 1 indication for the use of sotalol for the maintenance of sinus rhythm [5]. However, the use of sotalol for acute AF has not been recognised.

Although sotalol does not carry guideline recommendations, it is generally understood that guidelines are only guides, and not a substitute for clinical judgement. Many patients with AF have contraindications to the class 1 medications, but may not be willing to accept the side effect profile associated with amiodarone. This retrospective trial is observational and is not intended to examine the reasons why sotalol was chosen, but rather to acknowledge that medications are often used off guideline recommendations and provide additional information for physicians who are trying to provide reasonable treatment for $\mathrm{AF}$.

We sought to establish the efficacy and safety of oral sotalol as compared to other anti-arrhythmic drugs for acute $\mathrm{AF}$ in a district general hospital.

\section{Methods}

A retrospective analysis of 300 patients who were admitted with acute AF to a district general hospital between 1/1/2005-31/12/2005. Inclusion criteria included all patients considered to have primary AF. Exclusion criteria included patients who had acute AF requiring direct current cardioversion. Patients who had AF secondary to another cause such as sepsis, dehydration, alcohol, surgery, hyperthyroidism etc were also excluded.

Acute $\mathrm{AF} /$ recent onset $\mathrm{AF}$ was defined as $\mathrm{AF}$ of $<48$ hours duration [2] determined by the onset of patients symptoms. These patients were not known or documented to have AF previously. The type of drug administered was recorded i.e rate control or rhythm control. Rate control drugs included digoxin, betablockers, verapamil or diltiazem. Rhythm controlling agents prescribed included sotalol, amiodarone, flecanide, propafenone or disopyramide. Outcomes that were measured included all-cause mortality, cardiac death, readmission rates for $\mathrm{AF}$, congestive cardiac failure and all cause readmissions over a 2 year follow up period. Rates of cardioversion to sinus rhythm on discharge were noted.

\section{Statistical analyses}

Following a test of statistical normality (KomolgrovSmirnov test), data were expressed as mean with standard deviation (SD) or as median with interquartile range (IQR) for the normally distributed data and non-parametrically distributed data respectively. Comparisons between groups were analysed by 2-way repeated ANOVA or Kruskal-Wallis test, as appropriate. For paired data, the Wilcoxon matchedpairs signed-ranks or paired t-test were used. For unpaired data, Fisher's exact test was used. All analyses were performed using SPSS version 17.0. A p value of $<0.05$ was considered as statistically significant for all comparisons.

\section{Results}

Over the study period, 300 patients were admitted into the accident and emergency department with acute AF. drugs for each patient were documented (Table 1). One hundred and twenty patients received sotalol.

During admission 11 patients were treated with more than one drug. The maximum number of drugs used in any one patient was 3 agents. Twenty eight patients did not require any drug therapy as spontaneous cardioversion to sinus rhythm was achieved prior to arrival or whilst in the accident and emergency department. Patients who cardioverted to sinus rhythm prior to arrival to the accident and emergency department had an electrocardiogram performed by the paramedics initially confirming AF. Patients treated with sotalol received between $40 \mathrm{mg}-160 \mathrm{mg}$ twice daily orally, the majority (59 patients) receiving $80 \mathrm{mg}$ twice daily orally.

The remaining patients died predominantly of noncardiac causes. Mortality rates were lower in patients who received the higher dose of sotalol; $7.4 \%$ for patients who received $160 \mathrm{mg}$ BD versus $11.8 \%$ in those who received $80 \mathrm{mg} \mathrm{BD}$. However, this was not statistically significant. Compared to the rate control group, mortality rates were significantly lower in patients receiving an anti-arrhythmic drug; $29.8 \%$ versus $13.3 \%(\mathrm{p}<0.001)$ respectively. More specifically mortality rates were lower with sotalol when compared to all study drugs (Table 3).

\section{Cardio version to sinus rhythm on discharge and 2 year follow up}

Cardioversion to sinus rhythm on discharge 
was achieved in $68 \%$ of patients receiving an antiarrhythmic drug and $42 \%$ of patients receiving a rate limiting drug $(\mathrm{p}<0.001)$. This remained significant regardless of the anti-arrhythmic drug used. All patients in the anti- arrhythmic arm received one drug on discharge. Patients in the rate control group received between 1-3 drugs.

At 2 year follow up, sotalol $80 \mathrm{mg}$ BD had the highest cardioversion rates to sinus rhythm when compared to $40 \mathrm{mg} \mathrm{BD}$ and $120 \mathrm{mg} \mathrm{BD;78.0 \% ,67.6 \%} \mathrm{and} \mathrm{70.4 \%}$ respectively (Table 4 ).

Sotalol at any dose had a significantly higher cardioversion rate to sinus rhythm when compared to amiodarone $(\mathrm{p}=0.036)$ with a similar AF recurrence rate at 2 year follow up. Flecanide had the best cardioversion rate to sinus rhythm both on discharge and at 2 year follow up without any significant increase in complications.

\section{Readmission rates}

Overall readmission rates for recurrent AF was lower in patients treated with rate limiting drugs. Patients treated with sotalol or amiodarone had similar

\begin{tabular}{|cc|}
\hline Table 1: Discharge drugs for the 296 patients. \\
\hline Drugs & Number of patients \\
\hline Sotalol (40-160 mg BD) & 120 \\
\hline Amiodarone & 66 \\
\hline Flecainide & 16 \\
\hline Other anti arrhythmic drugs & 8 \\
\hline Rate Control medications & 75 \\
\hline No Drug Therapy & 28 \\
\hline
\end{tabular}

Table 2: Causes of death and mortality rates over a 2 year
follow up period.
\begin{tabular}{cc|} 
Cause of death & Number of patients \\
Cerebrovascular event & 11 \\
\hline Sudden death & 6 \\
\hline Congestive cardiac failure & 6 \\
\hline Acute coronary syndrome & 4 \\
\hline Other & 30 \\
\hline Death during first acute admission & 4 \\
\hline
\end{tabular}

readmission rates for recurrence of AF over a 2 year period (26\%).

\section{Discussion}

This study provides evidence for the safety and efficacy of sotalol in the pharmacological cardioversion of acute-onset/recent onset atrial fibrillation. The use of sotalol for recent $\mathrm{AF}$ is a class III indication and not recommended in the ACC/AHA/ESC 2006 guidelines for $A F$ as it is thought to be harmful [4]. In our hospital there was an approved use for sotalol and was used in patients who did not have any contraindications, such as those with a history of heart disease and patients who had a prolonged QTc interval or risk factors predisposing to arrhythmia.

The class III recommendation for the use of sotalol in acute AF was based on 3 studies [6-8]. Furthermore, all but one of the studies comprised of study populations of less than 100 patients. The larger cohort of patients (>100) in our study has provided insight regarding the use of sotalol in acute AF with favourable outcomes.

In this study, sotalol doses ranged between $80 \mathrm{mg}$ $-320 \mathrm{mg}$ daily with a mean total dose of $169.2 \mathrm{mg}$ daily. When divided into individual dosage groups there was no significant differences in outcomes. However, the limited sample size in each dosage group diminished the power of the statistical test. Cumulatively there was no evidence of a significant difference in either efficacy or mortality for doses of $160 \mathrm{mg}$ or less compared with doses of $240 \mathrm{mg}$ per day.

Cardioversion to sinus rhythm on discharge and after a 2 year follow up period was best achieved with flecanide, followed by sotalol at any dose and then amiodarone; $84 \%, 72 \%, 56 \%$ respectively. In a meta-analysis of 46 trials flecanide and ibutalide had the best evidence for the cardioversion of AF to sinus rhythm. Sotalol was found to have negative efficacy for cardioversion to sinus rhythm [9]. Though sotalol does exhibit some Vaughan Williams class III antiarrhythmic behavior, these effects are usually manifested at higher doses (180 mg and above). Only $23 \%$ of our patients

\begin{tabular}{|c|c|c|c|c|c|c|}
\hline & \multicolumn{3}{|c|}{ RHYTHM CONTROL ARM } & \multicolumn{3}{|c|}{ RATE CONTROL ARM } \\
\hline & Sotalol (120) & Amiodarone (66) & Other AAD (24) & Rate control (75) & No treatment (36) & \\
\hline Age & $68+/-8$ & $69+/-9$ & $61+/-8$ & $69+/-8$ & $68+/-6$ & \\
\hline SCD & $2(1.7 \%)$ & $2(3.0 \%)$ & $1(4 \%)$ & $3(4.0 \%)$ & 0 & \\
\hline All Cause Readmission & $67(56 \%)$ & $39(59 \%)$ & $12(50 \%)$ & $50(67 \%)$ & $22(61 \%)$ & \\
\hline Cardioversion to Sinus rhythm & $86(72 \%) p<0.0001$ & $37(56 \%) p<0.001$ & $20(83 \%) p<0.0001$ & $32(44 \%)$ & $15(42 \%)$ & \\
\hline
\end{tabular}




\begin{tabular}{|ccc|}
\hline Table 4: Cardioversion to sinus rhythm at 2 year follow up. \\
$\begin{array}{c}\text { Dumber of } \\
\text { patients }\end{array}$ & $\begin{array}{c}\text { Cardioversion to sinus } \\
\text { rhythm }\end{array}$ \\
\hline Sotalol $80 \mathrm{mg}$ daily & $23 / 34$ & $67.60 \%$ \\
\hline Sotalol $160 \mathrm{mg}$ daily & $46 / 59$ & $78 \%$ \\
\hline Sotalol $240 \mathrm{mg}$ daily & $19 / 27$ & $70.40 \%$ \\
\hline Amiodarone & $37 / 66$ & $56 \%$ \\
\hline Flecanide & $14 / 16$ & $88 \%$ \\
\hline All other AAD & $22 / 24$ & $92 \%$ \\
\hline Rate control drugs & $32 / 75$ & $42.70 \%$ \\
\hline
\end{tabular}

received the higher dose of sotalol.

In UK clinical practice, sotalol is often used at low doses (80-160 mg/day), at which it essentially acts in a similar manner to a class II beta adrenergic antagonist. The non-significant difference in the cardioversion rates between sotalol and amiodarone may well be due to the low dose of sotalol prescribed.

In another small study patients with acute AF were prescribed intravenous sotalol. There was no significant difference in cardioversion rates between intravenous sotalol and placebo [7] or between sotalol and amiodarone [10]. In these trials a single $1-1.5 \mathrm{mg} / \mathrm{kg}$ intravenous loading regime was prescribed as compared to the protracted oral regime in our study $[7,10,11]$.

In 33 patients who received a maximum dose of sotalol $320 \mathrm{mg}$ orally for acute AF the cardioversion rate to sinus rhythm was $52 \%$ compared to $86 \%$ taking quinidine. It is difficult to draw robust conclusions since there were a low number of patients that were recruited in this trial [12].

In our study there was no excess in mortality rates in patients who were prescribed sotalol. All-cause mortality and cardiovascular death rates were lower with sotalol when compared to patients treated with amiodarone or with rate limiting drugs. Previous studies have not highlighted areas of concern with regards to sotalol's safety [11-13].

The largest of the studies on sotalol included 103 patients in chronic AF/atrial flutter. Sotalol in this study was given intravenously, and no adverse outcome was observed despite rapid drug administration [11].

A small incidence of QTc prolongation without any adverse outcomes was seen when sotalol $320 \mathrm{mg}$ was given orally for persistent AF [6]. In a further trial of 61 patients, receiving a maximum dose of sotalol 320 mg orally for acute $\mathrm{AF}$, the discontinuation rate was $48 \%$ due to asymptomatic bradycardia or hypotension. Asymptomatic wide complex tachycardia was prevalent in $13 \%$ of patients [12].

When used as an antiarrhythmic agent, sotalol is often started at $80 \mathrm{mg}$ twice daily for the first week, and thereafter titrated to $160 \mathrm{mg}$ twice daily (or higher) after assessing the electrocardiogram for QT prolongation [10]. In our study patients were not prescribed sotalol if the QTc interval was prolonged or if there were electrolyte abnormalities which may have predisposed the patient to arrhythmia. Patients prescribed sotaolol subsequently had monitoring of their QTc interval by performing electrocardiograms during their follow up period. None of our patients had to discontinue sotalol due to QTc prolongation.

In a meta-analysis of 29 trials, sotalol and amiodarone were associated with adverse events and often required withdrawal [14]. In our study sotalol 40-160 mg BD was seen to be safe as there was no excess mortality.

Readmission rates for the recurrence of AF were 26\% for patients prescribed sotalol. Recurrence of $\mathrm{AF}$ with sotalol has been reported as $24 \%$ [6]. There is strong evidence for the maintenance of sinus rhythm with sotalol. The majority of trials assessing the recurrence of AF are based on an ECG at follow up or by ambulatory ECG monitoring. There are no trials assessing readmission rates to hospital with AF on sotalol. In the amiodarone arm readmission for recurrence $\mathrm{AF}$ was also $26 \%$. Amiodarone was found to be more effective at maintaining sinus rhythm at 1 year with a rate of $>65 \%$ when compared to sotalol and other anti-arrhythmic drugs [14-17].

It is important to note that the majority of AF trials with sotalol recruited a low number of patients or were done in patients who did not have acute AF but mainly had persistent AF.

The most recent guidelines, both the ACC/AHA/ HRS and the ESC guidelines on AF agree on the use of sotalol in patients without significant underlying heart disease (i.e., heart failure, coronary artery disease, or severe left ventricular hypertrophy) $[1,5]$.

Sotalol is a viable anti-arrhythmic option for the restoration of sinus rhythm in patients in whoem flecainide and amiodarone are contraindicated. Amiodarone side effect profile includes photosensitivity, abnormalities in thyroid and liver function tests as well as pulmonary fibrosis. In patients who are prescribed warfarin for $\mathrm{AF}$ the internationalised normalised ratio maybe affected with the concomitant use of Amiodarone. Therefore in such circumstances sotalol is a potential option. Furthermore in patients who have ischaemic heart disease and $\mathrm{AF}$ the dual action of rate control and rhythm control with sotalol is advantageous. Amiodarone however, is preferred in patients with $\mathrm{AF}$ who have had a previous myocardial infarction and heart failure [4]. Our study therefore provides evidence for the superior efficacy of sotalol for the management of acute AF in more than 100 patients.

\section{Conclusion}

This study examined a real world use of sotalol in an off label use in the management of acute onset AF 
and found it to be safe and effective. The combination of rate controlling properties with pharmacological cardioversion is attractive, especially in patients in whom flecanide and amiodarone is contraindicated. Sotalol demonstrated a significantly higher cardioversion rate on discharge and at 2 year follow up without an increase in mortality when compared to amiodarone and rate limiting drugs. Further studies in patients with acute atrial fibrillation are required to confirm our findings in a prospective manner and examine the optimum dosing regime.

\section{Executive summary}

- Objective: The European Society of Cardiology, American Heart Association and the American College of Cardiology guidelines on atrial fibrillation (AF) 2006 state that Sotalol should not be used in acute AF. We assessed the safety and efficacy of sotalol in acute AF when compared to other anti-arrhythmic drugs (ADD).

- Methods: A single centre retrospective observational study on 300 patients admitted with acute AF over a 12 months period. Study drugs used were sotalol, amiodarone, flecainide, propafenone or disopyramide for rhythm control. Digoxin, beta blockers, verapamil, diltiazem were prescribed for rate control. Rates of cardioversion to sinus rhythm, readmission rates due to $A F$, all cause readmissions, mortality rates due to sudden cardiac death and all-cause mortality was recorded over a 2 year follow up period. For paired data, the Wilcoxon matched-pairs signed- ranks or paired t-test were used. For unpaired data, Fisher's exact test was used.

- Results: 120 patients were discharged on sotalol. The mean total dose used was $169.2 \mathrm{mg}$ daily. Cardioversion to sinus rhythm on discharge occurred in $68 \%$ in the rhythm control group versus $42 \%$ for rate control group $(p<0.001)$. Sotalol had a significantly higher cardioversion rate regardless of the dose when compared to amiodarone $(p=0.036)$ however, there were similar readmission rates for AF. Four patients died acutely in hospital, none were on sotalol. Compared to all drugs sotalol had the lowest mortality rates $(p=0.001)$. Mortality rates were lower in patients who received the higher dose of sotalol; $7.4 \%$ for patients who received a total of $320 \mathrm{mg}$ daily versus $11.8 \%$ in those who received $160 \mathrm{mg}$ daily.

- Conclusion: Sotalol is as safe and effective as other anti-arrhythmic drugs; in fact it was significantly more effective than amiodarone in this cohort. All AAD's demonstrated a significant improvement in cardioversion rates and a significantly lower mortality rate than rate controlling drugs.

- The main presentations of these regular tachycardias are atrioventricular nodal re-entrant tachycardia and orthodromic atrioventricular reentrant tachycardia due to an accessory pathway.

\section{References}

1. Camm AJ, Lip GYH, De Caterina R, et al. 2012 focused update of the ESC guidelines for the management of atrial fibrillation. Eur. Heart. J. 33, 2719-2747 (2012).

2. Camm AJ, Kirchhof P, Lip GY, et al. Guidelines for the management of atrial fibrillation: the Task Force for the Management of Atrial Fibrillation of the European Society of Cardiology (ESC). Eur. Heart. J. 31, 2369-2429 (2010).

3. Wijffels MC, Kirchhof CJ, Dorland R, Allessie MA. Atrial fibrillation begets atrial fibrillation. A study in awake chronically instrumented goats. Circulation. 92, 1954-1968 (1995).

4. ACC/AHA/ESC Guideline committees. ACC/AHA/ESC 2006 guidelines for the management of patients with atrial fibrillation. J. Am. Coll. Cardiol. 48, 854-906 (2006).

5. January CT, Wann LS, Alpert JS, et al. 2014 AHA/ACC/ HRS Guideline for the management of patients with atrial fibrillation. J. Am. Coll. Cardiol. 64, e1-e76 (2014).

6. Hohnloser SH, Van de Loo A, Baedeker F. Efficacy and proarrhythmic hazards of pharmacologic cardioversion of atrial fibrillation: prospective comparison of sotalol versus quinidine. J. Am. Coll. Cardiol.26, 852-858 (1995).

7. Sung RJ, Tan HL, Karagounis L, et al. Intravenous sotalol for the termination of supraventricular tachycardia and atrial fibrillation and flutter: a multcenter, randomised, double- blind, placebo-controlled study. Am. Heart. J. 129, 739-748 (1995).

8. Thomas SP, Guy D, Wallace E, et al. Rapid loading of sotalol or amiodarone for management of recent onset symptomatic atrial fibrillation: a randomized, digoxin-controlled trial. Am. Heart. J. 147: E3 (2004).

9. McNamara RL, Bass EB, Miller MR, et al. Management of new onset atrial fibrillation. Evid. Rep. Technol. Assess. (Summ) 1-7 (2000).

10. Lip GYH, Apostolakis S. Atrial fibrillation (acute onset). BMJ. Clinical. Evidence. (Online) (2014).

11. Vos MA, Golitsyn SR, Stangl K, et al. Superiority of ibutilide (a new class III agent) over DL-sotalol in converting atrial flutter and atrial fibrillation. The Ibutilide/Sotalol Comparator Study Group. Heart. 79, 568-575 (1998).

12. Halinen MO, Huttunen M, Paakkinen S, Tarssanen L. Comparison of sotalol with digoxin-quinidine for conversion of acute atrial fibrillation to sinus rhythm (the SotalolDigoxin-Quinidine Trial). Am. J. Cardiol. 76, 495-498 (1995).

13. Singh S, Saini RK, DiMarco J, et al. Efficacy and safety of sotalol in digitalized patients with chronic atrial fibrillation. The Sotalol Study Group. Am. J. Cardiol. 68, 1227-1230 (1991). 
14. Freemantle N, Lafuente-Lafuente C, Mitchell S. Mixed treatment comparison of dronedarone, amiodarone, sotalol, flecainide, and propafenone, for the management of atrial fibrillation. Europace. 13, 329-345 (2011).

15. Le Heuzey JY, De Ferrari GM, Radzik D, et al. A short-term, randomized, double-blind, parallel-group study to evaluate the efficacy and safety of dronedarone versus amiodarone in patients with persistent atrial fibrillation: the DIONYSOS study. J. Cardiovasc. Electrophysiol. 21, 597-605 (2010).
16. Roy D, Talajic M, Dorian P, et al. Amiodarone to prevent recurrence of atrial fibrillation. Canadian Trial of Atrial Fibrillation Investigators. N. Engl. J. Med. 342, 913-920 (2000).

17. Singh BN, Singh SN, Reda DJ, et al. Amiodarone versus sotalol for atrial fibrillation. N. Engl. J. Med. 352, 1861-1872 (2005). 